\title{
Object Detection Algorithm Based on Moving Scene
}

\author{
Rong Zhou ${ }^{1, \text { a }}$, Qi Zhang ${ }^{1, b}$ \\ 'School of information Engineering Wuhan University of Technology Wuhan, 430070, China \\ a397232719@qq.com, b zhangqi6028@sina.com
}

\begin{abstract}
Keywords: Moving scene, object detection, Image registration, background generation Algorithm Abstract. This Intelligent video surveillance has been widely used in video surveillance environment, the traditional intelligent video surveillance algorithm has problem is that it is mainly for the static environment. It is can't detect the moving object in moving doom. So a new algorithm is proposed to expand the doom detect range by combine the moving detect algorithm with image registration algorithm. In the experiments, the algorithm can effectively expand the monitoring scene which can use in many circumvent.
\end{abstract}

\section{Introduction}

Intelligence video already extensively applied in various situation, be gradually also getting an application to the intelligence monitor of video frequency, however traditional video frequency intelligence monitor technique mainly aim at the background image is still, which limit the function of doom.

Moving object detect algorithm can classify into several method: frame-difference, the background generation[1], and optic-flows method etc. Along with the research, multi-algorithms are proposed, such as neural-network, SVM et.al [2].

Inter-frame difference method is adopted the subtraction operation of adjacent image. The algorithm has a disadvantage is that can't afford noise. Background generation algorithm has advantage of noise in actual surveillance environment. Several advantage algorithm are proposal to improve performance of background generation algorithm, Ming Han[3] put forward the improvement method through modify the parameter of background generation algorithm, which use multi-gauss model to adopt image noise. Ref[4,5] aimed at to improve adopt of image noise, through modify the parameter of threshold and background refresh.

The principle of these proposed algorithm base on a condition that scene is still. If scene changed or unstable will cause the moving target and background can't identify.

Image registration algorithm is widely used in many fields, such as aerial photography, which is mainly aimed at the process of continuous shooting. Richard Szeliski proposed a classic image mosaic algorithm based on image mosaic models[6], the algorithm can transform the image through the affine transformation of the relationship between different image matching, the image mosaic algorithm. With the deep research on image mosaic, many scholars have put forward several improved algorithms. Ref[7,8]proposed a fast algorithm for feature selection . Du Dong Mei[9]proposed a fast SURF algorithm for save memory space. The algorithm transform integral image into one image space for saving memory space, Zhu Qiguang[10] proposed using wavelet method, the image is divided into high frequency and low frequency part, in order to improve the extraction performance of the feature points, Zhang Zilong[11] in the selection of the feature points on the proposed new the algorithm, to achieve a more accurate feature points matching.

The traditional way of intelligent monitoring is to fix the scene of the dome camera and to detect the moving target. The more commonly used method is to use the background generation method, but the disadvantage is that the doom is fixed, which can't make the other direction Detection, restricted the use of doom. 
At present, the use of the dome's sport ability, to achieve a wider range of monitoring is an important application of the current direction, because the background generation algorithm itself defects, and difficult to meet.

Based on the above problems, a moving object detection algorithm based on non-fixed scene is proposed. Combined image registration algorithm with background generation algorithm, the background generation algorithm under the motion scene is constructed to improve the adaptability of the traditional moving object detection algorithm.

\section{Panorama Mosaic}

In this paper, we propose a new algorithm for the background generation algorithm, which is based on the background generation algorithm in the motion environment. The algorithm is shown in Figure 1 .

The algorithm is composed of background generation process and moving object detect process

The background generation process is shown as following steps:

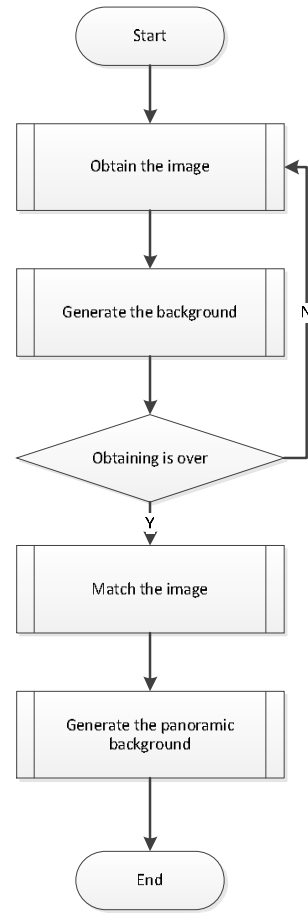

Figure 1 algorithm flow

1) Dome move to a position $i$, capture a serial of image $f_{i}$, generate background $B_{i}$ using the background generation algorithm ;

2) Move to the next position $j$, using the same process as step 1 generate background image $B_{j}$; while moving $\mathrm{N}$ time, obtain a set of background image $\left\{B_{0}, B_{1}, B_{2}, \ldots, B_{n}\right\}, n=N$;

3) Aiming at the sequence picture adoption picture to go together with quasi- calculate way to constitute is complete of background scene. According to ball machine current position $\mathrm{i}$, obtain a video frequency picture in a row, adopt a much Gauss background model method, set up a background picture;

4) Adopt sift calculate way, withdraw picture Cape to order an information, adopt RANSAC calculate way to match close together background $B_{n}, B_{n+1}$, In the RANSAC calculate way, the picture goes together with to acquire homologous projection transformation in the quasi- process matrix 


$$
w=\left[\begin{array}{l}
w x_{1} \\
w x_{2} \\
w x_{3}
\end{array}\right]=\left[\begin{array}{lll}
h_{1} & h_{2} & h_{3} \\
h_{4} & h_{5} & h_{6} \\
h_{7} & h_{8} & 1
\end{array}\right]\left[\begin{array}{l}
x_{1} \\
x_{2} \\
1
\end{array}\right]
$$

5) According to the transformation matrix merger picture, constitute an end panorama picture.

The advantage of the background composition algorithm is that it can construct a complete background image during the motion of the dome, which provides a basis for the subsequent detection of the moving object .this algorithm solve the difficult of tradition background generation algorithm.

\section{Exercise Object Examination}

The background object that obtains according to the last, during sport of the ball machine, passes to obtain a picture, realization to exercise the examination of target. Examine process in, need to complete three following steps.

Step 1.Matching of current moving scene

Step 2.moving object detection

Step 3.The background regeneration

Aim at three steps, we designed homologous sport object examination calculate way, the calculate way's process was as follows.

1) Get the image of the current position $f_{i}$, according to corresponding of $w_{i}$, transform to $f_{w_{i}}$, obtain the transformation's result represent as the rectangle $R_{i}=\left\{x_{1}, y_{1}, x_{2}, y_{2}\right\},\left\{x_{1}, y_{1}\right\}$ is the left top Cape of the picture , $\left\{x_{2}, y_{2}\right\}$ is the right bottom.

2) Transformation image $f_{w_{i}}$ and the panorama background image B mutually reduce and get a bad value picture $S_{i}=B-f_{w_{i}}, S \in R_{i}$.

3)Based on threshold extraction method, the corresponding difference threshold is obtained $T_{i}$.

4) While the image subtract result is bigger than $T_{i}$, which is the moving object, otherwise regeneration background according to coefficient $\gamma_{i}$.

5) The regeneration method is $B_{i+1}=B_{i} * \gamma_{i}+f_{w_{i}} *\left(1-\gamma_{i}\right)$.

Through the above steps, the detection of the moving target is completed and updated according to the corresponding update rules. 


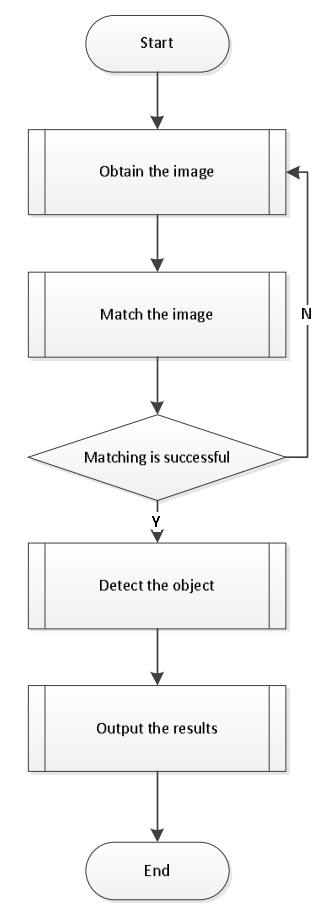

Figure 2 flow of moving object detect process

Through the above algorithm steps, it can be found that in the process of moving object detection, image registration is the core problem of background detection during the rotation of doom. The time consume of image registration process.

\section{Experiment}

Aiming at the algorithm proposed in this paper, the algorithm is designed and implemented with an actual scene. Experiment with the CPU at 2.3G, 4 nuclear, memory 4G, programing language is VC++.

The experiment of background generation process of algorithm as figure 3,4 show:

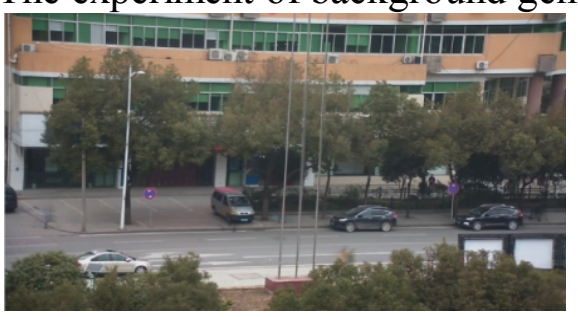

(1) Generation background picture 1

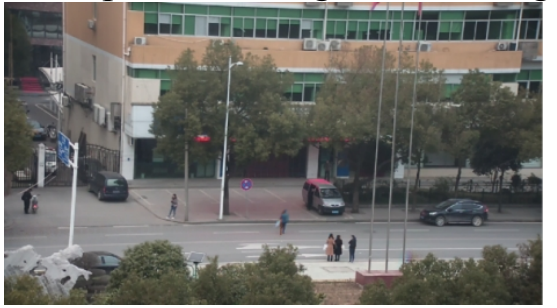

(2) Generation background picture 2

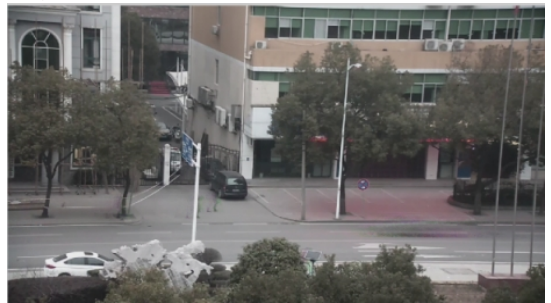

(3) Generation background picture 3

Figure 3 background generation

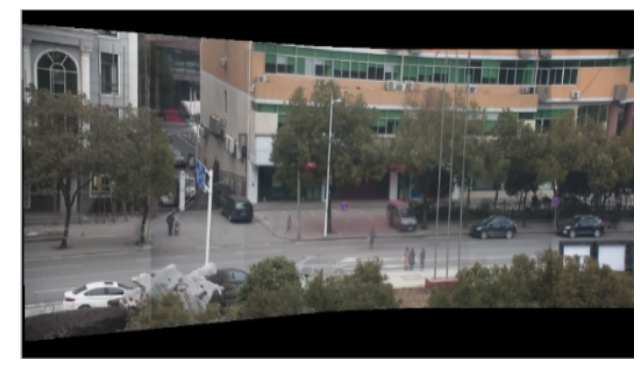

Figure 4: Panorama background scene

From the last diagram, after every background born process, set up sport scene examination background of integrity. 
The result of process of moving object detect show at figure 5:

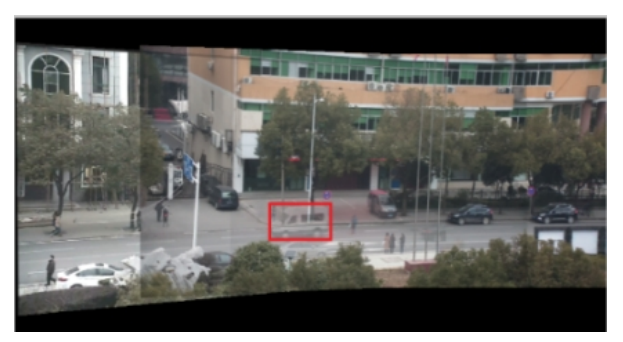

Figure 5: Exercise object examination

In order to evaluate the performance of the algorithm, we aim at calculate each main step. the performance of each step of the algorithm is show on table 1.

Table 1 computing performance

\begin{tabular}{lll}
\hline \multicolumn{1}{c}{ step } & Image size & time(s) \\
\hline Background generation & $1280 * 960$ & 1.2 \\
Panorama generation & & 1.3 \\
Image mosaic & $1280 * 960$ & 0.8 \\
Moving object & $1280 * 960$ & 0.5 \\
\hline
\end{tabular}

\section{Conclusions}

Through the above experiment, we can find that our method can be used to generate the background of the scene in the video, and the scenes in the process of the dome rotation are spliced to form a complete background .In the process of detecting the moving target, detection and extraction.

In this paper, the problems in the detection of moving objects are: the use of registration and background constantly updated, resulting a lower processing speed, the latter will focus on how to improve the existing image registration algorithm, Moving target detection speed, to achieve the purpose of real-time detection.

\section{References}

[1] Stauffer C;Grimson W E L.Adaptive background mixture models for real-time tracking[C].XIAO J Z, LEI B, WANG C Q. Reclamation on building waste produced from Wenchuan Earthquake[A].Computer Vision and Pattern Recognition, IEEE Computer Society Conference on, 1999.37-234.

[2] Zehang Sun;Bebis G.;Miller R. Monocular precrash vehicle detection: features and classifiers[J],IEEE Transactions on Image Processing,2006,5(7):2019-2025.

[3] Ming Han; Jiaomin Liu; Yi Sun.A Background Modeling Algorithm Based on Improved Adaptive Mixture Gaussian[J],Journal of Computers,2013,9(8):2239-2244.

[4] Qinghua $\mathrm{Lu}$, Zhiwei $\mathrm{Wu}$, Yanbin Fan. According to the sport vehicle examination method of hybrid Gauss model[J]. The photoelectron o laser, 2013,24(4):751-757.

[5] Huasheng Zhu, Jun Ye. According to improve the car discharge of Gauss model examination calculate way[J]. Laser with red outside, 2013,43(10):1180-1183. 
[6] Richard Szeliski. Video mosaics for virtual environments[J]. IEEE Computer Graphics and Applications, 1996.16(2): 22-30.

[7] Brown M, Lowe D G. Automatic Panoramic Image Stitching Using Invariant Features[J]. International Journal of Computer Vision, 2007, 74(1) : 59-73.

[8] Wu Y, Ma W, Gong M, et al. A Novel Point- matching Algorithm Based on Fast Sample Consensus for Image Registration $[\mathrm{J}]$. IEEE Geoscience and Remote Sensing Letters 2014,1(99),1-5.

[9] DU Dong-mei, WANG Hong. qi , TIAN Kun. pen, JIANG Leil,An Improved Fast SURF Algorithm, Science Technology and Engineering,2013,13(5),pp1350-1353.

[10] Zhu Qiguang, Wang Jia,Zhang Pengzhen, Chen Ying, Research on mobile robot localization based on Gaussian moment improved SURF algorithm, Chinese Journal of Scientific Instrument,2015,36(11),pp 2451-2457.

[11] Zhang Zilong;Xue Jing;Qiao Honghai;Zhi Yongfeng, The Vehicle Retrieval Methods of Traffic Video Based on Improved SURF Algorithm, Journal of Northwestern Polytechnical University,2014,32(2),pp 297-302. 